\title{
Metabolic syndrome and related dietary intervention among patients with coronary and peripheral arterial disease attending cardiovascular rehabilitation programs
}

\section{Sindrome metabolica ed intervento dietetico in pazienti con coronaropatia o arteriopatia periferica inclusi in programmi di riabilitazione cardiologica}

\author{
Marco Ambrosetti, Paola Mariani
}

\begin{abstract}
Metabolic syndrome and related dietary intervention among patients with coronary and peripheral arterial disease attending cardiovascular rehabilitation programs. M. Ambrosetti, P. Mariani.

Aim of the study: To provide estimates of the prevalence of metabolic syndrome (MS) among patients with coronary artery disease (CAD) or peripheral arterial obstructive disease (PAOD) attending cardiac rehabilitation (CR) programs, as well as related dietary needs targeted on the single core components of the syndrome.

Methods: Observational study enrolling 209 patients (males $75 \%$, mean age $65 \pm 8$ yrs.) referred to a CR facility because of silent ischemia $(11 \%)$, chronic stable angina $(28 \%)$, acute coronary syndrome $(\mathbf{4 1 \%})$, or peripheral arterial disease $(\mathbf{2 0 \%})$. The MS was diagnosed according to the 2005-modified NCEP ATP III criteria. Dietary regimens were classified into four areas of intervention (weight control, lipid control, glycaemic control, and
\end{abstract}

\begin{abstract}
blood pressure control) and compared in patients with and without MS.

Results: MS accounted for $26 \%$ of all patients, with the highest prevalence $(31 \%)$ among those admitted after acute coronary syndromes. All four dietary regimens were significantly more prescribed $(p<0.001)$ among patients with MS as compared to controls, with low sodium (95\%) and low fat $(\mathbf{9 0 \%})$ diets as the most represented patterns. All patients with MS were prescribed multiple dietary patterns, with adoption of a comprehensive low energy, low fat, and low glucose diet in up to one fifth of cases.

Conclusion: Patients with CAD or PAOD referred to CR programs often display an high cardiometabolic risk and need a broad regimen of dietary modification.

Keywords: metabolic syndrome, nutrition, coronary artery disease, peripheral arterial disease, cardiac rehabilitation.
\end{abstract}

Monaldi Arch Chest Dis 2007; 68: 227-230.

1 Division of Cardiology, IRCCS Fondazione S. Maugeri, Tradate, Italy.

2 Nutritional Services Unit - Health Care Department, IRCCS Fondazione S. Maugeri, Tradate, Italy.

Corresponding author: Marco Ambrosetti, MD; Cardiovascular Rehabilitation Unit, "Le Terrazze" Clinic; Via Ugo Foscolo, 6/b I-21035 Cunardo (VA), Italy; E-mail address: m.ambrosetti@clinicaleterrazze.com

\section{Introduction}

The significant burden of metabolic syndrome (MS) - a cluster of cardiovascular risk factors consisting of abdominal obesity, elevated blood pressure, elevated plasma glucose, and atherogenic dyslipidaemia - in the general population, as far as the strong association between MS and cardiovascular disease (CVD), give serious implications for health professionals in the field of cardiac rehabilitation (CR); understandably, MS deserves increased clinical attention as an associated comorbidity at the entry of rehabilitation programs, being patients qualified for a diagnosis of MS frequently candidates to more aggressive approach to management of cardiovascular risk factors. Conversely, physicians working in the CR setting often fail to recognize the importance to diagnose MS in patients with coronary artery disease (CAD) and peripheral arterial ob- structive disease (PAOD), this being likely due to the sparse body of evidence in this subgroup of patients: despite the large availability of communitybased studies, little evidence still exists on the prevalence of MS in patients referred for CR, as far as about their cardiovascular fate. Moreover, several agencies have stressed lifestyle intervention - weight reduction, anti-atherogenic diet, increased physical activity, and smoking cessation - as one way to consistently cut into the cardiovascular risk of patients with MS [1], but unfortunately a comprehensive strategy for appropriate management has not been definitely validated and offered as a guideline for CR health operators. Regarding dietary intervention, a growing body of research was originated in the field of obesity, hence focusing just on weight reduction and not encompassing all impaired components of the MS; the clustering of risk factors as a syndrome (and not simply the metabolic com- 
plications of obesity) reasonably allows to differentiate a structured diet for obesity from an hypothetical structured diet for MS. To begin to address these issues, we performed this study with the aim to provide estimates of the prevalence of MS in a representative CR unit and the global need for structured dietary intervention in these patients, as well as specific diet patterns targeted on the single core components of the MS.

\section{Methods}

\section{Study design}

In this observational study, a sample of patients referred to a cardiac rehabilitation facility in Northern Italy with a documented diagnosis of CAD or PAOD was tested for the presence of MS at admission. The sample was obtained at different times during the years 2004/2005, based on the availability of clinical information needed for the diagnosis of MS and documentation of nutritional evaluation. Only in-hospital patients were considered.

\section{Diagnosis of metabolic syndrome}

The clinical identification of metabolic syndrome was performed according to 2005-revised NCEP guidelines [1]. Patients were classified as having metabolic syndrome when three or more of the following risk factors were present: waist circumference $>102 \mathrm{~cm}$ and $88 \mathrm{~cm}$ in men and women respectively, triglycerides $\geq 150 \mathrm{mg} / \mathrm{dL}$, high-density lipoprotein cholesterol (HDL-C) $<40 \mathrm{mg} / \mathrm{dL}$ and 50 $\mathrm{mg} / \mathrm{dL}$ in men and women respectively, blood pressure $\geq 130 / \geq 85 \mathrm{mmHg}$, and fasting glucose $\geq 100$ $\mathrm{mg} / \mathrm{dL}$.

\section{Dietary intervention}

At the entry of the CR program, a balanced dietary program was appropriately designed by a dietitian in all patients. Current medical conditions targeting specific dietary modifications included obesity, dyslipidaemia, diabetes mellitus, and hypertension. Other modifications regarding certain nutrients (such as vitamin and mineral supplements) were assessed on individual basis. For the purposes of the study, dietary programmes were categorized into four main areas of intervention (weight control, lipid control, glycaemic control, and blood pressure control; table 1) according to medical needs shown by the single patient. However, the four dietary patterns were part of a comprehensive lifestyle modification, and shared several healthy food choices, such as the recommendation of maintaining an adequate intake of vegetables, fruits, and whole grains, as well as limiting alcohol consumption. The overall structure of the nutritional intervention also comprised delivering of intensive nutrition education by means of individual consultation at baseline and on demand, weekly group sessions, and provision of detailed written information.

\section{Statistical analysis}

Data were reported by means of descriptive statistics. Variables were examined by Student $t$ test, $\chi^{2}$ with Yates correction or Fisher exact tests where appropriate. A two-tailed p value $<0.05$ was considered statistically significant.

\section{Results}

The study population consisted of 209 patients (males $75 \%$, mean age $65 \pm 8$ yrs.) with the following reason for admission in the rehabilitation facility: silent ischemia in 23 patients (11\%), chronic stable angina in $58(28 \%)$, acute coronary syndrome in 87 $(41 \%)$, and peripheral arterial disease stage II according to Fontaine's classification in 41 (20\%). In $156(75 \%)$ cases a recent coronary or peripheral revascularization was performed. The reason for attending the CR program was a first manifestation of CVD among $80 \%$ of total cases.

Globally, the MS was diagnosed in 55 (26\%) patients, with the following prevalence of the core components of the syndrome: increased waist circumference $22 \%$, elevated triglycerides $18 \%$, decreased HDL-Cholesterol 44\%, elevated blood pressure $62 \%$, and increased fasting glucose $24 \%$. The prevalence of MS was highest (31\%) among patients admitted after acute coronary syndromes as compared to other cardiovascular conditions considered (figure 1).

The need for all single dietary patterns (figure 2) was significantly higher $(\mathrm{p}<0.001)$ in patients with MS as compared to controls, with low sodium (95\%) and low fat (90\%) diets as the most represented patterns. Focusing on low fat, low calories, and low glucose diets (i.e. the more structured dietary interventions), a proportion of $34.3 \%$ of the total amount of diets delivered was prescribed to patients with MS (figure 3); of these, $28.7 \%$ was prescribed to patients with indication to two coexisting dietary patterns, and $5.6 \%$ to patients in whom all three dietary patterns were indicated. Among patients with MS, the prescription of a low energy diet against obesity always needed to be integrated by other dietary patterns, with low sodium, low fat, and low glucose diets accounting for $92 \%, 87 \%$, and $37 \%$ of cases respectively.

Table 1. - Indication to different dietary treatments among CAD/PAOD patients referred to cardiac rehabilitation

\begin{tabular}{ll}
\hline Dietary treatment & Indication \\
\hline Caloric restriction & $-\mathrm{BMI}>25$ and/or \\
& $-\mathrm{WC}>102(\mathrm{M})$ and $88(\mathrm{~F}) \mathrm{cm}$ \\
\hline Controlled lipid intake & - LDL-cholesterol $>100 \mathrm{mg} / \mathrm{dl}$ and $/ \mathrm{or}$ \\
& - HDL-cholesterol $<40(\mathrm{M})$ and $<50(\mathrm{~F}) \mathrm{mg} / \mathrm{dl}$ and/or \\
& - Triglycerides $>150 \mathrm{mg} / \mathrm{dL}$ \\
\hline Controlled carbohydrate intake & - Fasting plasma glucose level $>100 \mathrm{mg} / \mathrm{dL}$ \\
\hline Sodium restriction & - Blood pressure $>140 / 90 \mathrm{mmHg}($ or $>130 / 85$ if \\
& patient has diabetes $)$ \\
\hline
\end{tabular}

CAD: coronary artery disease; PAOD: peripheral arterial obstructive disease; BMI: body mass index; WC: waist circumference; M: males; F: females; LDL: low-density lipoprotein; HDL: high-density lipoprotein. 


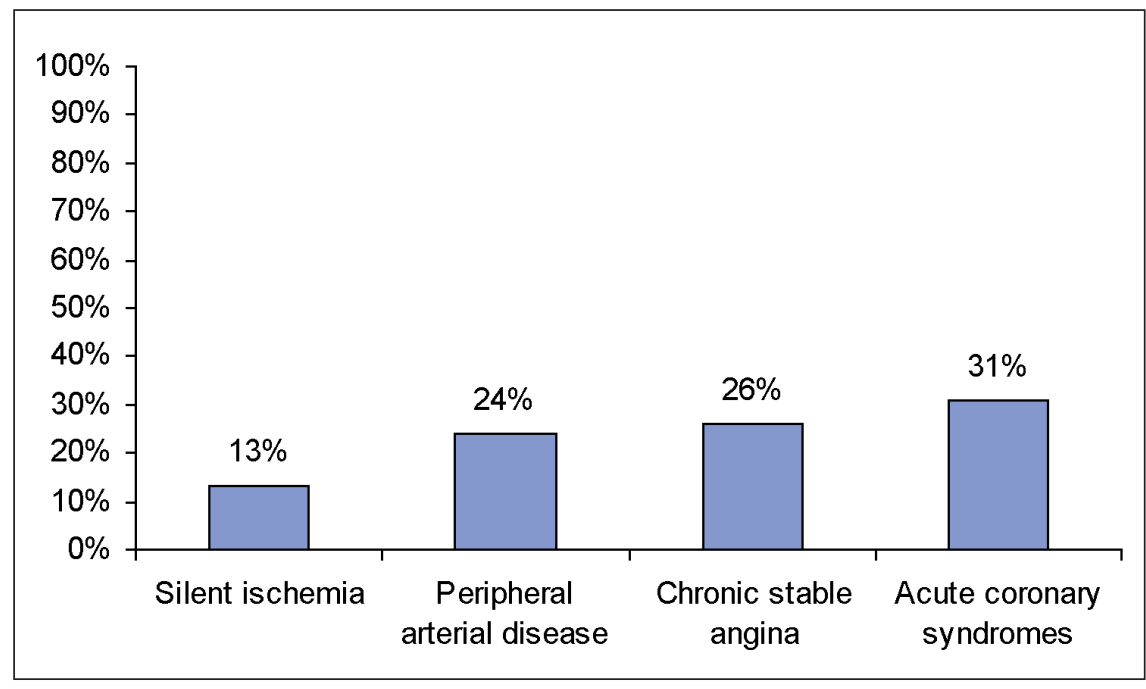

Figure 1. - Prevalence of metabolic syndrome among patients admitted for cardiac rehabilitation according to different diagnoses.

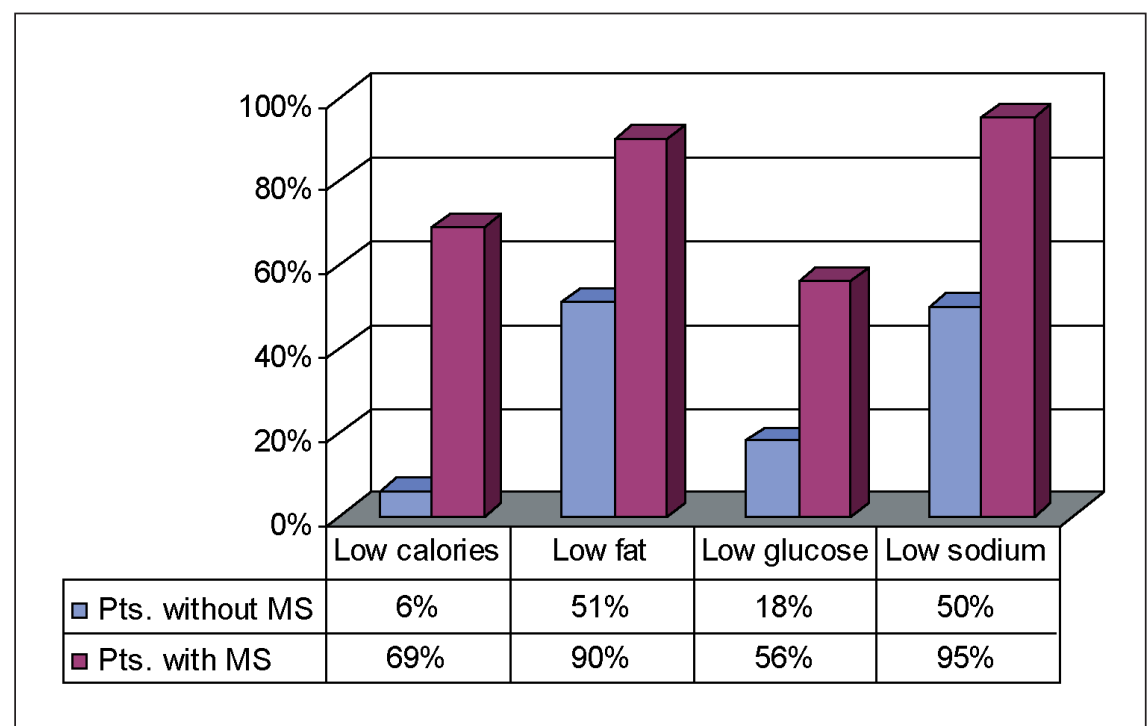

Figure 2. - Dietary patterns according to the presence of metabolic syndrome (MS). All differences were statistically significant with a $\mathrm{p}<0.001$.

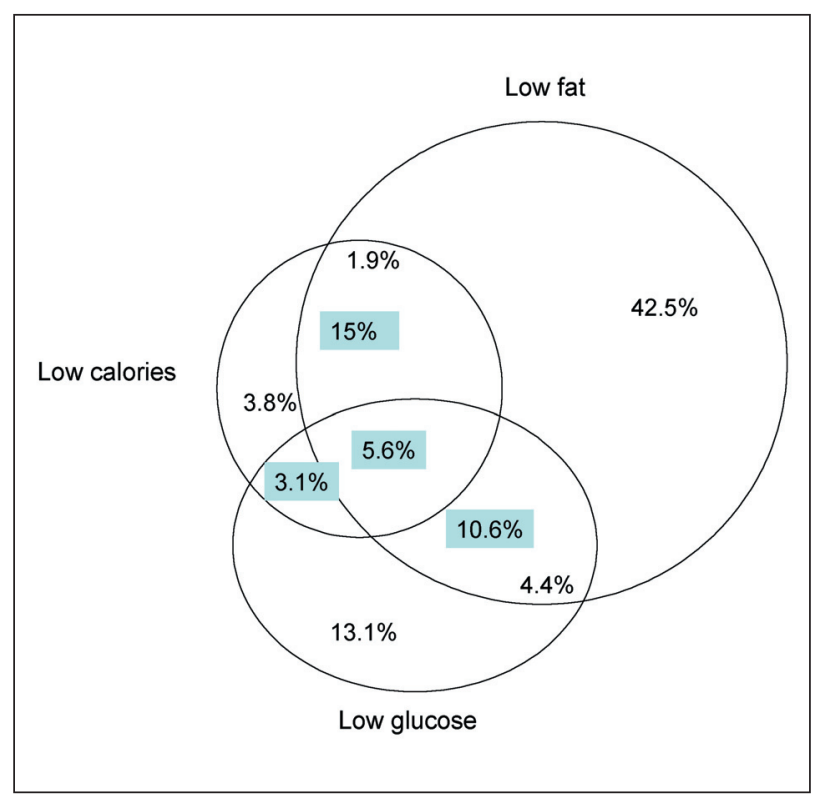

Figure 3. - Distribution of dietary treatment regimens according to individual needs. Marked percentages are referred to the population of pts. with metabolic syndrome.

\section{Discussion}

In this report, we address two questions: the presence of MS among different subgroups of patients at the entry of CR programs, and the magnitude of the effort aimed at ensuring an appropriate medical nutrition therapy.

Based on our data, the MS is a frequent coexisting comorbidity among patients attending CR programs. We are aware of only two publications $[2,3]$ specifically addressing the presence of MS in the CR setting, both derived from studies performed in the U.S.A., and reporting a cumulative prevalence of about $50-60 \%$. Of particular note, the study by Savage et al. [3] provided evidence for considering MS as closely associated to the whole population of patients referred to CR, rather than a condition confined to selected manifestations of CVD. These findings could be interpreted as an argument for actively searching the presence of MS when evaluating the global cardiovascular risk of our patients.

Nutritional counselling is considered of greatest importance for successful secondary prevention programmes. Since the increased emphasis on the high prevalence of MS in the population referred to $\mathrm{CR}$ services, we focused on dietary needs of these patients and found that, despite the overlap between MS and obesity, a more integrated dietary approach should be advocated rather than just controlling body weight. It could also be noted that these patients are often de novo prescribed nutritional therapy (as confirmed by the high proportion of first CVD manifestation in our sample), and consequently the opportunity to significantly reduce the risk of future events by means of intensive lifestyle modification should not be missed.

Based on current evidence, the exciting question as to whether a specific diet for patients with MS could be identified is likely to remain unsolved. An elegant review by Baxter et al in 2006 [4] found only three large studies on dietary patterns and the MS. Data from population-based cross-sectional studies showed that dietary patterns high in fruit and vegetables were associated with reduced prevalence of MS, as well as a diet high in fish; conversely, a significant relationship between protein from animal sources and MS was found, while plant proteins showed no association; finally, diets with a high intake of cereals and dairy foods gave inconclusive results. Among cereal-based 
foods, minimally processed cereal foods - in contrast to processed cereals - showed a lower risk of insulin resistance and obesity, while the hypothesis that a selected dairy food intake could give protection against MS required further confirmation. Regarding fat intake, data from the multicenter KANWU study [5] on 162 healthy subjects revealed that decreasing saturated fatty acid and increasing monounsaturated fatty acid might improve insulin sensitivity without effect on insulin secretion; however, authors stated that the beneficial effect of monounsaturated fat disappeared when total fat intake exceeded $38 \%$ of total energy [6]. Taken together, these findings suggest that diet composition could affect insulin resistance - the dominant pathophysiological pattern of MS - and could be harmonized in a structured dietary program to be investigated by controlled clinical trials.

In conclusion, our study highlighted the elevated cardiometabolic risk carried out by a large proportion of patients attending CR programs, as well as the need for a structured cardiometabolic-protective eating pattern, driven by all core components of MS displayed by the single patient.

\section{Riassunto}

La sindrome metabolica (SM) è presente in circa un terzo dei 209 pazienti con cardiopatia ischemica o arteriopatia ostruttiva degli arti inferiori partecipanti ad un programma di Riabili- tazione Cardiologica. Tutti i regimi dietetici (ipocalorico, ipolipidico, ipoglicemico, anti-ipertnsivo) sono stati utilizzati più frequentemente nei pazienti con SM. Pertanto i pazienti con SM richiedono interventi dietetici multicomprensivi diretti ad affrontare le singole componenti della sindrome.

\section{References}

1. Grundy SM, Cleeman JI, Daniels SR, et al. American Heart Association; National Heart, Lung, and Blood Institute. Diagnosis and management of the metabolic syndrome: an American Heart Association/National Heart, Lung, and Blood Institute Scientific Statement. Circulation 2005; 112: 2735-2752.

2. Milani RV, Lavie CJ. Prevalence and profile of metabolic syndrome in patients following acute coronary events and effects of therapeutic lifestyle change with cardiac rehabilitation. Am J Cardiol 2003; 92: 50-54.

3) Savage PD, Banzer JA, Balady GJ, Ades PA. Prevalence of metabolic syndrome in cardiac rehabilitation/secondary prevention programs. Am Heart $J$ 2005; 149: 627-631.

4. Baxter AJ, Coyne T, McClintock C. Dietary patterns and metabolic syndrome - a review of epidemiologic evidence. Asia Pac J Clin Nutr 2006; 15: 134-142.

5. Vessby B, Unsitupa M, Hermansen K, et al. Substituting dietary saturated for monounsaturated fat impairs insulin sensitivity in healthy men and women: The KANWU Study. Diabetologia 2001; 44: 312-319.

6. Riccardi G, Rivellese AA. Dietary treatment of the metabolic syndrome - the optimal diet. Br J Nutr 2000; 83: S143-148. 